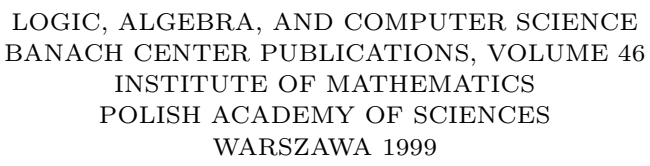

\title{
DECIDABILITY AND STRUCTURE
}

\author{
PAWE€ M. IDZIAK \\ Computer Science Department \\ Jagiellonian University \\ Nawojki 11 \\ 30-072 Kraków, Poland \\ E-mail:idziak@ii.uj.edu.pl
}

An important and fundamental area of research in Mathematical Logic has been an attempt to classify and understand those structures or collections of structures that have decidable theories. A structure is said to be decidable if there is an algorithm to decide precisely which sentences (taken from a language appropriate for the structure) are true of the structure.

The study of the decision problem for various classes of structures began in the 1930's when Church gave the first undecidability result.

In Algebra one primarily studies collections of algebras known as varieties (that is classes of algebras closed under subalgebras, products and homomorphic images, or equivalently classes defined by a set of equations). Over past few decades there has been a systematic attempt to classify decidable varieties.

Soon after the appearance of the undecidability result of A. Church and J. B. Rosser, namely that Peano's arithmetic is undecidable, A. Tarski took over and developed in 1938-39 a general framework for proving the undecidability of first-order theories. The method was used by himself and many others to obtain a wide spectrum of undecidable varieties, including

(u.1) multi-unary algebras,

(u.2) (non-Abelian) groups,

(u.3) Heyting algebras, monadic algebras,

(u.4) (distributive) lattices,

(u.5) semilattices, semigroups.

1991 Mathematics Subject Classification: 03D35, 08B10, 08B25.

Work supported by Polish KBN grant 2P03A-031-09.

The paper is in final form and no version of it will be published elsewhere. 
Thus from the very beginning it was clear that a rich enough mathematical theory should be undecidable. On the other hand A. Tarski [33] proved (in 1949) the decidability of the class of Boolean algebras. Another important result of this kind is the one of W. Szmielew ([32], 1955), saying that any variety of Abelian groups is decidable. These, together with the decidability of algebras with one unary operation (the decidability of which was shown by A. Ehrenfeucht [5], 1959) form, as it turned out recently, the paradigms for decidable varieties. Although the proofs of undecidability often can be performed without any finiteness assumptions on a variety $\mathcal{V}$ one can hardly expect to get an effective (or, according to Hilbert, a finitinistic) method without any such restriction. It should not be surprising that we restrict ourselves to

- locally finite varieties, i.e. varieties in which every finitely generated algebra is finite,

or sometimes even to

- finitely generated varieties, i.e. classes of the form $\operatorname{HSP}(\mathcal{K})$, where $\mathcal{K}$ is a finite set of finite algebras.

Another important assumption we make is that

- varieties are of finite type, i.e. there are only finitely many fundamental operations.

Note that the above mentioned basic examples of decidable varieties, i.e.

(d.1) mono-unary algebras,

(d.2) Abelian groups,

(d.3) Boolean algebras,

are of finite type.

All of the results we had mentioned so far answer the question of whether a particular variety is decidable. However there were no general theorems concerning the structure of decidable varieties. The situation changed in the middle of 1970's when H. Werner [38] extended a sheaf technique, used earlier by S. D. Comer [4] for monadic algebras, to prove that each finitely generated discriminator variety has a decidable first order theory.

The easiest way to see how a discriminator variety looks like is to imagine it as consisting of Boolean algebras with extra operators. Instead of giving a precise definition of a discriminator variety let us make the following digression.

We know that each variety $\mathcal{V}$ is generated by its subdirectly irreducible algebras, i.e., $\mathcal{V}=\operatorname{HSP}\left(\mathcal{V}_{S I}\right)$ is the smallest class containing all subdirectly irreducibles from $\mathcal{V}$ and closed under formation of homomorphic images, subalgebras and direct products. In fact each algebra $\mathbf{A}$ can be embedded into a product of SI-algebras:

$$
\mathbf{A} \subseteq \prod_{t \in T} \mathbf{B}_{t}
$$

in a way that each projection $\pi_{t}: A \longrightarrow B_{t}$ is surjective. (By a subdirectly irreducible algebra we mean one that cannot be further 'decomposed' in this way, or equivalently an algebra having a least non-trivial congruence.) In general such kind of embedding does 
not tell us much how the algebra $\mathbf{A}$ lies inside the product $\prod_{t \in T} \mathbf{B}_{t}$. Therefore only a few things can be said about a particular subdirect embedding. However, sometimes it is possible to transfer a big deal of information from the stalks $\left\{\mathbf{B}_{t}\right\}_{t \in T}$ to a subdirect product $\mathbf{A}$. This happens when the index set $T$ is endowed with some extra structure usually including a topological one - and $\mathbf{A}$ is uniquely determined by this structure of $T$ and $\left\{\mathbf{B}_{t}\right\}_{t \in T}$.

This is the case when considering discriminator varieties. Each algebra from such a variety can be represented by the so called Boolean product of the SI's (which are actually simple, i.e., have no proper congruences). In Boolean products the index set $T$ is endowed with Stone topology (i.e. $T$ is a totally disconnected Hausdorff compact space). If $\mathcal{V}$ is finitely generated then every algebra $\mathbf{A} \in \mathcal{V}$ is determined uniquely by (and therefore can be recovered from) its congruence lattice $\operatorname{Con}(\mathbf{A})$ labeled by the class $\mathcal{V}_{S I}$. The facts that the last class is finite and Stone spaces are just duals of Boolean algebras allowed H. Werner [38] effectively translate sentences about algebras from $\mathcal{V}$ into sentences about Boolean algebras. Since the last class is decidable then we get

THEOREM 1 (Werner [38]). Every finitely generated discriminator variety is decidable.

As we will see later discriminator varieties are the only congruence distributive ones (i.e. such that the congruence lattice $\operatorname{Con}(\mathbf{A})$ is distributive whenever $\mathbf{A} \in \mathcal{V}$ ) that can be decidable.

The class of congruence distributive varieties is broad enough to include Boolean algebras, Heyting algebras or more generally most algebras with lattice operations. This covers many varieties that arise as algebraic counterparts of (non-)classical logics. However the only varieties of classical algebraic structures that are congruence distributive are those generated by finite fields.

In the 70's A. P. Zamyatin $[42,43,44,45]$ gave a complete characterization of decidable varieties of groups and rings. Excellent results of S. D. Comer, H. Werner and A. P. Zamyatin were used by S. Burris and R. McKenzie ([1], 1981) to describe the structure of decidable locally finite congruence modular varieties. Among congruence modular algebras there are all congruence distributive ones as well as almost all classical algebraic structures like groups, rings, modules, quasi-groups and many others. An important role is played here by affine varieties, i.e. varieties $\mathcal{A}$ for which there is a $\operatorname{ring} R$ such that every algebra $\mathbf{A} \in \mathcal{A}$ is polynomially equivalent to some $R$-module.

Theorem 2 (Burris, McKenzie [1]). A locally finite congruence modular variety $\mathcal{V}$ is decidable if and only if there is a decidable affine variety $\mathcal{A}$ and a decidable discriminator variety $\mathcal{D}$ such that $\mathcal{V}$ decomposes into $\mathcal{V}=\mathcal{A} \otimes \mathcal{D}$.

The decomposition in the last theorem means that every algebra $\mathbf{V} \in \mathcal{V}$ can be uniquely represented as a product $\mathbf{V}=\mathbf{A} \times \mathbf{D}$ with $\mathbf{A} \in \mathcal{A}$ and $\mathbf{D} \in \mathcal{D}$.

In the 80 's R. McKenzie and M. Valeriote [24] went further to drop even the modularity assumption. In their decomposition, besides a discriminator variety and an affine one there is a third factor: a combinatorial (or, strongly Abelian) variety, i.e. a variety $\mathcal{S}$ that 
satisfies the following term condition:

$$
\mathbf{t}\left(a, u_{1}, \ldots, u_{k}\right)=\mathbf{t}\left(b, v_{1}, \ldots, v_{k}\right) \text { implies } \mathbf{t}\left(a, w_{1}, \ldots, w_{k}\right)=\mathbf{t}\left(b, w_{1}, \ldots, w_{k}\right)
$$

whenever $\mathbf{t}$ is a term and $a, b, u_{1}, \ldots, u_{k}, v_{1} \ldots, v_{k}, w_{1}, \ldots, w_{k} \in \mathbf{S} \in \mathcal{S}$. For example any variety in which all operations are unary (or depend only on a single variable) are combinatorial.

Theorem 3 (McKenzie, Valeriote [24]). A locally finite variety $\mathcal{V}$ is decidable if and only if there is a decidable combinatorial variety $\mathcal{S}$, a decidable affine variety $\mathcal{A}$ and a decidable discriminator variety $\mathcal{D}$ such that $\mathcal{V}$ decomposes into $\mathcal{V}=\mathcal{S} \otimes \mathcal{A} \otimes \mathcal{D}$.

Theorem 3 does not actually answer the question which locally finite (or even finitely generated) varieties are decidable. It reduces the problem to combinatorial, affine and discriminator varieties.

The combinatorial case has been completely solved by M. Valeriote in [34], where it is shown that decidable combinatorial varieties are those that are equivalent to multisorted unary algebras satisfying certain linearity condition. Much less is known about which affine or discriminator varieties are decidable.

Discriminator varieties have nice representation via sheaves not only when finitely generated (cf. Theorem 1). Using sophisticated sheaf constructions S. Burris, R. McKenzie, M. Valeriote [3] along with R. Willard [37, 39, 40], have recently made significant progress in the study of decidable discriminator varieties.

For results on decidable affine varieties the reader may consult the papers $[25,26,27$, 28, 29] of F. Point and M. Prest.

There are two big achievements in the join effort of Burris, McKenzie and Valeriote (Theorems 2 and 3). First there is an algorithm that converts a finite algebra $\mathbf{A}$ (of finite type) into a finite ring $R$ such that the variety $\operatorname{HSP}(\mathbf{A})$ generated by $\mathbf{A}$ is decidable if and only if the variety of modules over the ring $R$ is decidable.

Second, the proofs of those results give a deep insight into the structure of decidable varieties. This was made possible by two big developments of the late 70's and early 80 's. One of them is modular commutator theory. The theory had been initiated by J. D. H. Smith [31] for congruence permutable varieties. It was further developed by J. Hagemann and Ch. Herrmann [8], H. P. Gumm [7] and R. Freese and R. McKenzie [6]. The last book contains several important results and techniques that are extremely useful when studying congruence modular varieties. The binary operation on congruences that simultaneously generalizes the concept of a commutator $[H, K]$ of two normal subgroups $H, K$ of a group as well as the ideal multiplication in rings was defined. Then it was shown how some information about algebras or varieties can be recovered from congruence lattices endowed with this binary operation. Moreover the concept of commutator allows us to speak about solvable, nilpotent or Abelian congruence (or algebra) as well as about the center of an algebra or a centralizer of a congruence.

The second achievment of Universal Algebra was tame congruence theory created and described in D. Hobby and R. McKenzie [9]. Tame congruence theory is a tool to study the local structure of finite algebras. Instead of considering the whole algebra and all its operations at once, tame congruence theory allows us to localize to small subsets 
on which the structure is much simpler to understand and handle. There are only five possible ways a finite algebra can behave locally. It can be either one of the following:

1. a finite set with a group action on it,

2. a finite vector space over a finite field,

3. a two element Boolean algebra,

4. a two element lattice,

5. a two element semilattice.

Now, if a local behavior of an algebra is 'bad' then we can often show that the algebra itself behaves 'badly'. For example, since distributive lattices and semilattices are (finitely) undecidable then one can argue that in a (finitely) decidable variety structures of type 4 or $\mathbf{5}$ cannot occur.

On the other hand it is not true that if the local behavior is 'nice' then the global one is such. Several kinds of interactions between these small sets can produce a fairly messy global behavior. Such interactions often contribute to (finite) undecidability. Also relative 'geographical layout' of those small sets can result in unpredictable phenomena.

Theorem 3 was obtained by detecting almost 20 different ways in which a 'bad' local behavior can occur. In every such situation the hereditarily undecidable class $\mathcal{G}_{\text {fin }}$ of finite graphs (see [23]) was uniformly interpreted into $\mathcal{V}$. The interpretation (or semantical embedding) of an undecidable class is a standard technique for proving undecidability (see [2] or [1]). And often this undecibable class is the class $\mathcal{G}_{\text {fin }}$. Thus from the proof of Theorem 3 we know that if a semantical embedding of $\mathcal{G}_{\text {fin }}$ into a locally finite variety $\mathcal{V}$ is not possible then $\mathcal{V}$ has a nice structure. This leads to the following definitions.

A class $\mathcal{V}$ is said to be

- unstructured if the class $\mathcal{G}_{\text {fin }}$ can be semantically embedded into $\mathcal{V}$,

- finitely unstructured if the class $\mathcal{G}_{\text {fin }}$ can be semantically embedded into $\mathcal{V}_{\text {fin }}$.

The reader should be warned here that there are (finitely generated) undecidable varieties that are not unstructured. Modules (over a finite ring) provide a natural example. They have a stable theory and therefore cannot interpret graphs. Also R. Willard [41] provides an example of a finitely undecidable locally finite discriminator variety that is not known to be finitely unstructured.

From Theorem 3 we know that decidable varieties are really quite rare. Essentially we have three kinds of them. Locally the structure of factors from $\mathcal{S}, \mathcal{A}$ and $\mathcal{D}$ is of type 1, 2 and 3, respectively. And each of these three kinds of decidable varieties slightly generalizes those listed in (d.1)-(d.3).

Decidable varieties were characterized in terms of the behavior of congruences of their algebras. Birkhoff's suggestion in the 1930's that congruence lattices should be considered as fundamental associated structures has proved to be remarkably farsighted. In particular, the mentioned results of S. Burris, R. McKenzie and M. Valeriote justified an old standing supposition that decidability of a variety and its nice structure theory go hand in hand. In case a variety is finitely generated and decidable then all its algebras can be recovered, up to isomorphism, from their (labeled) congruence lattices and a single finite algebra. 
The connections between the structure of algebras and behavior of their congruences is extremely strong if one restricts to the class of finite algebras. This leads to the question of finite decidability of a given class of algebras - a class is said to be finitely decidable if the first order theory of its finite members is recursive. Although many of undecidable varieties are also finitely undecidable, these two notions are completely independent any of the four possible cases can occur. Boolean algebras are both decidable and finitely decidable while distributive lattices are undecidable and finitely undecidable. From the work of A. P. Zamyatin [45] it follows that the variety of rings generated by the ring of integers modulo 4 is undecidable but finitely decidable. The most interesting example was found by J. Jeong [19] who constructed a decidable discriminator variety that is finitely undecidable.

For a better understanding how such differences can occur we will consider the following two examples:

- a finitely decidable theory of one equivalence relation, i.e. the theory of the class $\mathcal{E}_{1}$ of all structures $(X ; \rho)$ with $\rho$ being an equivalence relation on a finite set $X$,

- a finitely undecidable theory of two equivalence relations, i.e. the theory of the class $\mathcal{E}_{2}$ of all structures $\left(X ; \rho_{1}, \rho_{2}\right)$ with $\rho_{1}, \rho_{2}$ being equivalence relations on a finite set $X$,

One can show that the second class is finitely unstructured. Thus if $\mathcal{E}_{2}$ can be semantically embedded into finite algebras from a variety $\mathcal{V}$ then one can conclude that $\mathcal{V}$ itself is finitely unstructured (and therefore finitely undecidable). An easy example of such situation occurs in the class of finite Boolean triples, i.e. structures of the form $\left(\mathbf{B} ; B_{1}, B_{2}\right)$, where $\mathbf{B}$ is a finite Boolean algebra and $B_{1}, B_{2}$ are two unary predicates distinguishing subalgebras of $\mathbf{B}$; simply take $\mathbf{B}$ to be the Boolean algebra on power-set $2^{X}$ and $B_{i}$ to be the subalgebra of $\mathbf{B}$ consisting of unions of $\rho_{i}$-equivalence classes. Now we replace $B_{i}$ by a unary closure function $c_{i}$, i.e. for $Z \subseteq X$ we let $c_{i}(Z)$ to be the smallest subset in $B_{i}$ that contains $Z$. This allows us to pass to the finitely undecidable variety of cylindric algebras (of dimension 2).

Actually it happens quite often that finite Boolean triples (or in other words two equivalence relations) 'live locally' in finite algebras from a variety. Two such general situations were detected while proving:

THEOREM 4 (Idziak $[12,13])$. Every finitely decidable locally finite congruence distributive variety is congruence permutable and congruence linear.

Here congruence permutability means that the relational product of congruences is commutative. It is a strong condition. For example it implies that every subalgebra $\mathbf{D}$ of $\mathbf{A} \times \mathbf{A}$ that contains the diagonal $\{(a, a): a \in A\}$ is symmetric (as a binary relation on $A)$. This seriously restricts the global behavior of finite algebras in $\operatorname{HSP}(\mathbf{A})$.

Congruence linearity is the condition saying that congruences in subdirectly irreducible algebras (from $\mathcal{V}$ ) are linearly ordered. This gives that the set $\mathrm{Cm}(\mathbf{A})$ of meetirreducible congruences of a (finite) algebra from $\mathcal{V}$ is a dual tree under the inclusion, i.e. for $\alpha, \beta, \gamma \in \mathrm{Cm}(\mathbf{A})$

$$
\alpha \subseteq \beta, \gamma \text { gives } \beta \subseteq \gamma \text { or } \gamma \subseteq \beta \text {. }
$$


For example, if we consider (finite) monadic algebras (i.e. cylindric algebras of dimension 1) that are obtained from $\mathcal{E}_{1}$ (in a way analogous to the dimension 2 case), then meetirreducible congruences of the algebra corresponding to $(X ; \rho)$ form a tree (or forest) isomorphic to the disjoint union $X \cup X / \rho$ with the membership relation $\in$ as the ordering.

Now given this tree $\mathrm{Cm}(\mathbf{A})$ one can expect that the very strong result of M. O. Rabin [30] on decidability of the Monadic Second Order theory of finite trees can be applied to get the decidability of $\mathcal{V}_{\text {fin }}$. One can see how this can be done in case of the variety $\mathcal{L C}$ of Heyting algebras generated by all chains (see [10] for details). For every Heyting algebra $\mathbf{A}$ from $\mathcal{L C}$ the set of meet-irreducible elements form a tree $T_{\mathbf{A}}$. The universe of a finite $\mathbf{A} \in \mathcal{L C}$ can be recovered as the collection $O\left(T_{\mathbf{A}}\right)$ of all subsets of $T_{\mathbf{A}}$ that are closed upwards. Moreover the operations of $\mathbf{A}$, when applied to $O\left(T_{\mathbf{A}}\right)$, are expressible in terms of set-theoretic operations of subsets of $T_{\mathbf{A}}$, and therefore can be defined in the Monadic Second Order language of trees. This together with Rabin's result gives the finite decidability of $\mathcal{L C}$.

In general we cannot expect that (finite) algebras from $\mathcal{V}$ are recoverable just from their trees of meet-irreducible congruences. Nevertheless P. M. Idziak [11] succeeded in defining a special kind of sheaf construction and in representing finite algebras in an appropriate way (see also $[14,15])$ to prove the converse of Theorem 4 in finitely generated setting:

THEOREM 5 (Idziak). A finitely generated congruence distributive variety (of finite type) is finitely decidable if and only if it is congruence permutable and congruence linear.

Soon after the above results had been published J. Jeong applied Tame Congruence Theory to extend them to congruence modular varieties. After proving that

- every finitely decidable locally finite congruence modular variety is congruence permutable (see [20]),

he analyzed subdirectly irreducible algebras in such varieties $\mathcal{V}$. In particular he showed (see [21]) that the monolith $\mu$ of a finite subdirectly irreducible algebra $\mathbf{A} \in \mathcal{V}$ has the following properties:

- the centralizer $(0: \mu)$ of $\mu$ is an Abelian congruence,

- the congruences of $\mathbf{A}$ above $(0: \mu)$ are linearly ordered,

- the algebra $\mathbf{A} /(0 ; \mu)$ together with all its homomorphic images has no non-trivial Abelian congruences.

The most important consequence of these facts is that

- any finitely decidable finitely generated congruence modular variety has only finitely many subdirectly irreducible algebras and all of them are finite.

Another necessary condition of this kind was found by P. M. Idziak and M. Valeriote $[17]$ :

- the centralizer $(0: \mu)$ of the monolith $\mu$ of a subdirectly irreducible algebra $\mathbf{A} \in \mathcal{V}$ is comparable to all congruences of $\mathbf{A}$. 
All of the above conditions were obtained by interpreting $\mathcal{G}_{\text {fin }}$ into $\mathcal{V}_{\text {fin }}$, so that actually we have:

TheOREM 6 (Jeong; Idziak, Valeriote). If a finitely generated congruence modular variety $\mathcal{V}$ is not finitely unstructured then

(1) $\mathcal{V}$ is congruence permutable,

$(2) \mathcal{V}$ has only finitely many subdirectly irreducible algebras and all of them are finite

(3) if $\mathbf{A}$ is a subdirectly irreducible algebra in $\mathcal{V}$ and $\nu$ is the centralizer of the monolith of $\mathbf{A}$, then

(3.1) $\nu$ is an Abelian congruence,

(3.2) $\nu$ is comparable with all congruences of $\mathbf{A}$,

(3.3) the congruences of $\mathbf{A}$ above $\nu$ are linearly ordered,

(3.4) $\mathbf{A} / \nu$ has no homomorphic image with Abelian monolith.

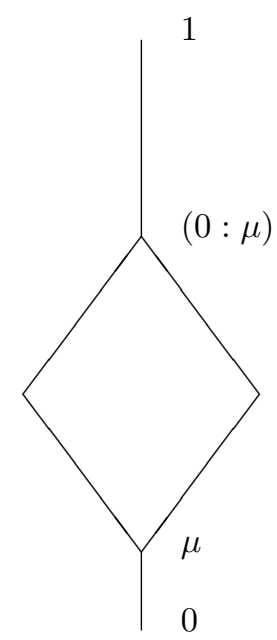

Theorem 6 lists several situations in which a 'bad' local behavior in a (finite) subdirectly irreducible algebra propagates into members of $\mathcal{V}_{\text {fin }}$ in a way that allows a uniform embedding of $\mathcal{G}_{\text {fin }}$.

In particular those conditions separate an Abelian part (below the centralizer $(0: \mu)$ ) and a nonAbelian part (above the centralizer $(0: \mu)$ ) in a congruence lattice $\operatorname{Con}(\mathbf{A})$ of a subdirectly irreducible algebra. Moreover they describe the nice (linear) behavior of the non-Abelian part (see the picture).

Since the Abelian (or affine) part does not allow interpretation of graphs we cannot expect any further conditions on the behavior of the congruences below the centralizer $(0: \mu)$ in varieties that are not finitely unstructured. What is more interesting is that the list from Theorem 6 is complete. If $\mathcal{V}$ satisfies all those conditions then $\mathcal{V}_{\text {fin }}$ has 'nice' structure.

To get an impression how this structure is obtained we start with noticing that in every finite algebra $\mathbf{B}$ there is the largest solvable congruence $\rho$, called the solvable radical of $\mathbf{B}$. Our conditions from Theorem 6 guarantee that this radical is in fact Abelian. This in turn allows to endow the product of $\rho$-cosets with the module structure. In particular we get a finite family $\mathcal{R}=\left\{R_{\mathbf{A}}: \mathbf{A} \in \mathcal{V}_{S I}\right\}$ of finite rings such that the product of $\left(0: \mu_{\mathbf{A}}\right)$-cosets of a subdirectly irreducible algebra $\mathbf{A}$ is an $R_{\mathbf{A}}$-module.

Another consequence of our theorem is that congruences of $\mathbf{B}$ that lie above the radical $\rho$ form a distributive lattice in which the set

$$
T_{\mathbf{B}}=\{\varphi \in \mathrm{Cm}(\mathbf{B}): \varphi \supseteq \rho\}
$$

is a (dual) tree under inclusion. This dual tree is the main 'frame' from which we will 
recover B. Obviously to do that we need to know more than just the tree $T_{\mathbf{B}}$. This knowledge is encoded by a double labeling of $T_{\mathbf{B}}$.

The first labeling associates with every $\varphi \in T_{\mathbf{B}}$ a subdirectly irreducible algebra (from a previously fixed list) that is isomorphic to $\mathbf{B} / \varphi$. To do this in a uniform way we first create a large 'amalgam' $\mathbf{D}$ of subdirectly irreducibles from $\mathcal{V}$. Then we define a monotone mapping

$$
\Theta: T_{\mathbf{B}} \ni \varphi \mapsto \Theta_{\varphi} \in \mathrm{Cm}(\mathbf{D})
$$

that among other compatibility conditions satisfies $\mathbf{B} / \varphi \cong \mathbf{D} / \Theta_{\varphi}$.

The second label for the node $\varphi$ is a module $M_{\varphi}$ over the ring $R_{\mathbf{D} / \Theta_{\varphi}}$.

In [16] a substantial extension of a construction from [11] was defined. This construction associates with arbitrary collection of the form $(\mathbf{D}, T, \Theta, M)$ where

- $\mathbf{D}$ is an algebra,

- $T$ is a partially ordered set,

- $\Theta: T \longrightarrow \mathrm{Cm}(\mathbf{D})$ is a monotone mapping,

- $M=\left\{M_{t}\right\}_{t \in T}$ is a family of modules

that satisfies certain compatibility conditions, an algebra

$$
\mathbf{D}[T, \Theta, M]
$$

in the variety $\mathrm{HSP}(\mathbf{D})$ generated by $\mathbf{D}$. This construction was shown to be good enough to represent every finite algebra $\mathbf{B}$ from the variety $\mathcal{V}$ as $\mathbf{D}\left[T_{\mathbf{B}}, \Theta, M\right]$, where $\Theta_{\varphi}$ and $M_{\varphi}$ are the two labelings of $T_{\mathbf{B}}$ defined earlier. Thus we have:

THEOREM 7 (Idziak [16]). Let $\mathcal{V}$ be a finitely generated congruence modular variety. If $\mathcal{V}$ is not finitely unstructured then there is a finite algebra $\mathbf{D}$ and a finite collection $\mathcal{R}_{\mathcal{V}}$ of finite modules such that finite algebras from $\mathcal{V}$ are exactly those of the form $\mathbf{D}[T, \Theta, M]$, where $T$ is a finite dual tree, $\Theta: T \longrightarrow \mathrm{Cm}(\mathbf{D})$ is a monotone mapping and $M$ is a family of modules over rings from $\mathcal{R}_{\mathcal{V}}$.

The construction and representation described in Theorem 7 allows us to apply a Feferman-Vaught style analysis. This in turn effectively reduces the first order theory of the class $\mathcal{V}_{\text {fin }}$ to the monadic second order theory of finite trees and the first order theory of finite modules over a finite number of finite rings. Thus we get

THEOREM 8 (Idziak [16]). For every finitely generated congruence modular variety $\mathcal{V}$ (of finite type) there is a finite family of finite rings $\mathcal{R}_{\mathcal{V}}$ (effectively computable from any finite generator of $\mathcal{V})$ such that $\mathcal{V}$ is decidable if and only if $\mathcal{V}$ is not finitely unstructured, and for every $R \in \mathcal{R}_{\mathcal{V}}$ the class of all $R$-modules is finitely decidable.

Much work has been done in a more general setting, i.e. without the congruence modularity assumption. Results of Idziak, Jeong, Valeriote and Willard [17, 18, 22, 35, 36] allow us to hope that analogues of Theorems 6,7 and 8 may hold. It is natural to expect that in this general setting we have to incorporate structures that are 'locally combinatorial' or unary.

The research on (finite) decidability of varieties demonstrated the power and usefulness of the algebraic tools: the Commutator Theory and the Tame Congruence Theory. 
Those tools allow to talk about algebras or varieties that are completely unrestricted in nature except that they satisfy certain finiteness conditions.

\section{References}

[1] S. Burris and R. MCKenzie, Decidability and Boolean Representation, Memoirs Amer. Math. Soc., 246(1981).

[2] S. Burris and H. P. Sankappanavar, A Course in Universal Algebra, Springer Verlag 1981.

[3] S. Burris, R. McKenzie and M. Valeriote, Decidable discriminator varieties from unary varieties, Journal of Symbolic Logic, 56(1991), 1355-1368.

[4] S. D. Comer, Elementary properties of structures of sections, Bol. Soc. Mat. Mexicana, 19(1974), 78-85.

[5] A. Ehrenfeucht, Decidability of the theory of one function, Notices Amer. Math. Soc., 6(1959), 268.

[6] R. Freese and R. MCKenzie, Commutator theory for congruence modular varieties, London Math. Soc. Lecture Notes, 125, 1987.

[7] H. P. Gumm, Geometrical methods in congruence modular varieties, Memoirs Amer. Math. Soc., 289(1983).

[8] J. Hagemann and C. Herrmann, A concrete ideal multiplication for algebraic systems and its relation to congruence distributivity, Archive der Mathematik, 32(1979), 234-245.

[9] D. Hobby and R. McKenzie, The Structure of Finite Algebras, Amer. Math. Soc., Contemporary Mathematics Volume 76, Providence, 1988.

[10] K. Idziak and P. M. Idziak, Decidability problem for finite Heyting algebras, Journal of Symbolic Logic, 53(1988), 729-735.

[11] P. M. IDZIAK, Reduced sub-powers and the decision problem for finite algebras in arithmetical varieties, Algebra Universalis, 25(1988), 365-383.

[12] P. M. Idziak, Varieties with decidable finite algebras I: linearity, Algebra Universalis, 26(1989), 234-246.

[13] P. M. IDZIAK, Varieties with decidable finite algebras II: permutability, Algebra Universalis, 26(1989), 247-256.

[14] P. M. IDZIAK, Sheaves in universal algebra and model theory, Part I, Reports on Mathematical Logic, 23(1989), 39-65.

[15] P. M. Idziak, Sheaves in universal algebra and model theory, Part II, Reports on Mathematical Logic, 24(1990), 61-86.

[16] P. M. IDZIAK, A characterization of finitely decidable congruence modular varieties, Trans. Amer. Math. Soc., 349(1997), 903-934.

[17] P. M. Idziak and M. Valeriote, A property of the solvable radical in finitely decidable varieties. manuscript 1992.

[18] P. M. IDZiaK and M. VAleriote, The centralizer in finitely decidable varieties. manuscript 1996.

[19] J. Jeong, On finitely decidable varieties, Ph.D. Thesis, Univ. California, Berkeley, 1991.

[20] J. JEOnG, Finitary decidability implies permutability for congruence modular varieties, Algebra Universalis, 29(1992), 441-448.

[21] J. Jeong, Finitely decidable congruence modular varieties, Trans. Amer. Math. Soc., 339(1993), 623-642. 
[22] J. JeOng, Type 2 subdirectly irreducible algebras in finitely decidable varieties, Journal of Algebra, 174(1995), 772-793.

[23] I. A. LAVROV, Effective inseparability of the sets of identically true formulae and finitely refutable formulae for certain elementary theories, (Russian) Algebra i Logika, 2(1963), $5-18$.

[24] R. McKenzie and M. Valeriote, The Structure of Decidable Locally Finite Varieties, Birkhäuser, Boston, 1989.

[25] F. Point, Problèmes de décidabilité pour les théories de modules, Bull. Soc. Math. Belg. Ser. B, 38(1986), 58-74.

[26] F. Point, Decidability questions for theories of modules, Proc. of Logic Colloquium '90 (Helsinki, 1990), Lecture Notes in Logic, vol. 2, pp. 266-280, Springer, Berlin, 1993.

[27] F. Point and M. Prest, Decidability for theories of modules, J. London Math. Soc. (2), 38(1988), 193-206.

[28] M. Prest, Model theory and modules, London Math. Soc. Lecture Notes, 130, 1988.

[29] M. Prest, Wild representation type and undecidability, Comm. Algebra, 19(1991), 919929.

[30] M. O. Rabin, Decidability of second order theories and automata on infinite trees, Trans. Amer. Math. Soc., 141(1969), 1-35.

[31] J. D. H. Smith, Malcev Varieties, Lectures Notes in Mathematics, vol.554, Springer Verlag 1976.

[32] W. Szmielew, Elementary properties of Abelian groups, Fund. Math., 55(1955), 203-271.

[33] A. TARski, Arithmetical classes and types of Boolean algebras, Bull. Amer. Math. Soc., 55(1949), 64.

[34] M. Valeriote, On decidable locally finite varieties, Ph.D. Thesis, Univ. California, Berkeley, 1986

[35] M. VALERIOTE, On solvable congruences in finitely decidable varieties, Mathematical Logic Quarterly, 40(1994), 398-414.

[36] M. Valeriote and R. Willard, Some properties of finitely decidable varieties, International Journal of Algebra and Computation, 2(1992), 89-101.

[37] M. Valeriote and R. Willard, Discriminating varieties, Algebra Universalis, 32(1994), $177-188$.

[38] H. Werner, Discriminator Algebras, Studien zur Algebra und ihre Anwendungen, Band 6, Akademie-Verlag, Berlin 1978.

[39] R. Willard, Decidable discriminator varieties from unary classes, Trans. Amer. Math. Soc., 336(1993), 311-333.

[40] R. Willard, Decidable discriminator varieties with lattice stalks, Algebra Universalis, 31(1994), 177-194.

[41] R. Willard, Hereditary undecidability of some theories of finite structures, Journal of Symbolic Logic, 59(1994), 1254-1262.

[42] A. P. Zamyatin, A prevariety of semigroups whose elementary theory is solvable, Algebra and Logic, 12(1973), 233-241.

[43] A. P. Zamyatin, Varieties of associative rings whose elementary theory is decidable, Soviet Math. Dokl., 17(1976), 996-999.

[44] A. P. Zamyatin, A non-Abelian variety of groups has an undecidable elementary theory, Algebra and Logic, 17(1978), 13-17.

[45] A. P. Zamyatin, Prevarieties of associative rings whose elementary theory is decidable, Soviet Math. Dokl., 19(1978), 890-901. 